Thorax (1954), 9, 71.

\title{
A CLINICAL, RADIOGRAPHIC, AND PATHOLOGICAL STUDY OF PULMONARY EMBOLISM
}

\author{
BY \\ J. G. MACLEOD AND I. W. B. GRANT \\ From the Departments of Medicine and Respiratory Diseases, University of Edinburgh
}

(RECEIVED FOR PUBliCATION MARCH 30, 1953)

This study of 60 cases of pulmonary embolism was undertaken in an attempt to elucidate the pathological changes produced in the lungs and in the pulmonary circulation in such cases and to correlate these with their clinical and radiographic manifestations. The two aspects of the subject which seemed to demand particular attention were the diversity of the effects of pulmonary embolism and the difficulty in reconciling the variable and often transient clinical and radiographic features of pulmonary infarction with the usual pathological conception of that condition, haemorrhagic pulmonary necrosis. This study is concerned primarily with the effects of pulmonary embolism, and the events preceding embolism will be discussed only in so far as they relate to subsequent developments. The results of the study are presented in two parts. First an account is given of the clinical and radiographic findings and thereafter the pathological interpretation is presented in the light of these findings and with reference to relevant experimental and other work.

\section{CLINICAL AND RADIOGRAPHIC FINDINGS}

In the course of a period of approximately two years over 100 cases of pulmonary embolism were seen in the medical, surgical, gynaecological and obstetric wards of the Western and Eastern General Hospitals, Edinburgh. Of these, only 60 were considered suitable for detailed study. In the others, either the quality of the radiographs was poor, many of the films having perforce to be taken with a mobile apparatus, or radiographic examination had not been made sufficiently often for adequate observation of the course of the disease.

Of the 60 cases studied, two were suffering from the effects of massive pulmonary embolism when first seen. These were the only cases of this type included in the series as all the others died before radiographic examination could be undertaken. The two cases were of considerable interest radiographically and pathologically, and will be considered separateiy. The remaining 58 presented the features of pulmonary infarction.

A diagnosis of pulmonary infarction can be made with certainty only at necropsy. During life it must depend on circumstantial evidence. It would have been possible to avoid presumptive diagnosis by confining the study to fatal cases. Additional material could then have been obtained by studying in retrospect cases in which the condition was first recognized post mortem. This policy was not adopted for two reasons. In the first place it was believed that clinical and radiographic observation of the non-fatal cases might provide information relevant to the pathology of pulmonary infarction that could not be obtained from the fatal cases alone. Secondly, a retrospective clinical or radiographic study of cases not recognized during life would almost certainly have been incomplete or unreliable. Accordingly it was decided to observe both fatal and non-fatal cases in which the diagnosis of pulmonary infarction was made during life, while admitting the possibility of inaccurate diagnosis in a few of the nonfatal cases.

In pulmonary infarction there is no single manifestation, clinical or radiographic, that cannot occur in most other types of pulmonary disease. In practice, however, a diagnosis of pulmonary infarction can usually be made when pleuritic pain, haemoptysis, or both, occur either in association with a known source for pulmonary emboli, or in circumstances known to predispose to thrombo-embolism such as prolonged recumbency, a recent major surgical operation, or a recent confinement. Further support is given to the diagnosis if the onset of symptoms suggesting pulmonary infarction is immediately preceded by an episode of acute dyspnoea, central chest pain, or circulatory collapse, such as might be produced by a fairly large embolus lodging in the pulmonary trunk or in one of its major branches. 
As one of the purposes of the investigation was to elucidate the nature of the radiographic changes in pulmonary infarction, the results of this examination were not allowed to influence the clinical diagnosis, except to exclude other diseases. It would, in fact, have been difficult to adopt any other course as, at the time the investigation was begun, we were unable, either in the light of personal experience or from a study of the literature, to formulate any reliable criteria for a radiographic diagnosis of pulmonary infarction.

\section{Analysis of Cases of Pulmonary Infarction}

The number of cases in the series is too small for detailed statistical analysis. Such an analysis in respect of predisposing circumstances and of symptoms such as pain and haemoptysis would also be invalidated by the fact that these features have been employed as diagnostic criteria. The analysis which follows is thus intended to convey mainly a general impression of the aetiological factors, the clinical and radiological features, the course, the prognosis, and the complications in a group of cases of pulmonary infarction.

\section{Aetiological factors in Pulmonary Infarction}

SEX INCIDENCE.-The 58 cases consisted of 29 males and 29 females. This proportion supports the view of most observers that the disease is equally common in the two sexes.

Circumstances of OnSET.-Twenty-five cases occurred after a surgical or gynaecological operation and four after parturition. Twenty-nine cases, belonging to neither of these categories, are referred to as "medical cases." Most of these medical cases developed pulmonary infarction while under treatment in hospital for some other disease, but in four cases this was not so, pulmonary infarction itself being the reason for admission to hospital. In each of these four cases the infarction was preceded by venous thrombosis in a lower limb occurring when the patient was ambulant and apparently in good health.

Relationship to Heart Disease.-Of the 58 cases of pulmonary infarction, 17 had organic heart disease and 41 had not (Table I). Of the 17 , rheumatic heart disease was present in nine, and hypertension or coronary disease in the remainder. Heart disease was encountered in just over half of the "medical" cases, but in only two of the 29 post-operative and post-partum cases.

AGE INCIDENCE.-Approximately two-thirds of the patients were between 40 and 70 years old. The average age, 55 years, was the same in both post-operative and "medical" cases and also in $\overrightarrow{\bar{N}}$ all cases with, and in all cases without, heart disease.

TABLE I

RELATIONSHIP TO HEART DISEASE

\begin{tabular}{cc|cc|c}
\hline \multicolumn{2}{c|}{ Category of Case } & $\begin{array}{c}\text { With } \\
\text { Heart Disease }\end{array}$ & $\begin{array}{c}\text { Without } \\
\text { Heart Disease }\end{array}$ \\
\hline Post-operative & $\ldots$ &. & 2 & 23 \\
Post-partum & $\ldots$ & $\ldots$ & 0 & 4 \\
"Medical" & . & $\ldots$ & 15 & 14 \\
\hline Total &. &. & 17 & 41 \\
\hline
\end{tabular}

SOURCE OF EMBOLUS.-As in most other reports on the subject, the commonest source was a lower limb vein $(60 \%$ of all cases in this series). There was a higher proportion of cases in the heart disease group in which the source of the embolus $z$ was not identified. It may well be that in some of these cases the pulmonary infarction was due, not $\stackrel{5}{工}$ to embolism, but to primary pulmonary arterial $\vec{\oplus}$ thrombosis. Alternatively, the infarction may on have been the result of embolism secondary to intra-cardiac thrombosis, for example, from auricular fibrillation which was present in six of the 11 cases in which the source of the embolus was not identified.

\section{Clinical Features of Pulmonary Infarction}

In 52 of the 58 cases only one incident of pul- 3 monary infarction was observed. In five cases there were two separate incidents weeks or months? apart and in one case there were three such incidents. The survey of clinical features which follows is based on the total of 65 incidents. As $\underset{\times}{\times}$ no important differences were noted in the clinical $\frac{0}{3}$ features of the post-operative, the post-partum, and the "medical" cases, an analysis has beeno made only in respect of the presence or absence of heart disease.

Pleuritic Pain.-This was present in 58 of the $>$ 65 incidents, giving a total incidence of $90 \%$. It was usually the presenting symptom. The pain was on the right side in 33 cases, on the left in $16^{\circ}$ cases, and on both sides in nine cases. In seven $\mathcal{N}^{-}$ cases there was no pain. There was no demon- $\omega$ strable difference in the incidence or distribution of pain between the cardiac and non-cardiace groups.

HAEMOPTYSIS. - Haemoptysis was present in $32^{?}$ incidents (just under half) and absent in the remainder. It was seldom severe and often took the form of bloodstained sputum being expec- $\frac{\rho}{\bar{D}}$ torated over a period of several days. Haemop- $\stackrel{\mathbb{Q}}{\Omega}$ tysis was more common in patients with pulmonary congestion secondary to heart disease. 
Cough AND Sputum.-These (apart from sputum associated with haemoptysis) were absent in more than two-thirds of all incidents. Frequently, when these symptoms were present, they antedated the pulmonary infarction and probably had some other cause. The incidence of cough and sputum was the same in the cardiac and non-cardiac groups. We came to the conclusion that the absence of cough and sputum lent some support to a diagnosis of pulmonary infarction.

Other Clinical Features.-Certain other clinical features were seen.

Dyspnoea was a variable symptom and difficult to assess as it was so often associated with pleuritic pain. It seemed to bear little relationship to the size and number of the infarcts.

Pyrexia was variable from case to case. Most had a low fever of between $99^{\circ}$ and $100^{\circ} \mathrm{F}$. for three to seven days, but in some there was no pyrexia and in others the temperature rose, usually in the first 24 to 48 hours, to $102^{\circ}$ or higher. In a few cases there was low pyrexia persisting for one to four weeks. There was no recognizable correlation between the degree of pyrexia and the extent of the infarction.

Tachycardia.-The heart rate was above 100 per minute in the majority of incidents. Usually it was abnormally rapid in proportion to the degree of pyrexia, and in two of the seven incidents in which there was no pyrexia it exceeded 120. In general, however, it helped little in diagnosis.

Cyanosis was present only in cases with very extensive infarction and in cases with heart failure.

Icterus, which Short (1952) observed in many cases, was not noted in this series except in patients with long-standing congestive cardiac failure, in whom it was ascribed to cardiac cirrhosis.

Physical Signs in the Chest.-These were seldom gross. The commonest abnormality was the finding of coarse or medium crepitations, generally in association with diminished air entry. In a few cases the physical signs of consolidation were present. In several patients physical signs suggesting pleural effusion were elicited, but in many of these instances a subsequent radiograph showed that the signs were due, not to effusion, but to a high hemidiaphragm. In some cases the pleural rub and the radiographic changes were on opposite sides. This observation was considered of importance as it appeared to indicate that in some cases an infarct could be recognizable clinically, by the pleural rub, and yet produce no radiographic changes.

Changes in Blood Count.-In most cases of pulmonary infarction examination of the blood showed a polymorph leucocytosis. A common figure was 12,000 per c.mm., but total white counts of up to 17,000 per c.mm. were recorded even in the absence of evidence of secondary infection. Significant degrees of anaemia were not observed.

Character of Pleural Liquid.-Pleural liquid was obtained for examination in only four cases, as it did not seem justifiable to submit patients with small effusions to the discomfort of needling. In all four cases the liquid was serous in character, and the predominant cells were polymorphs and the protein content was above $4 \%$.

Cardiac Failure.-Congestive cardiac failure quite frequently preceded the development of pulmonary infarction. In other cases the infarction appeared to precipitate congestive cardiac failure. Both these events were observed only in cases with pre-existing heart disease. Acute cor pulmonale developed in two cases of pulmonary infarction in which massive embolism occurred as a terminal event. No cases of chronic cor pulmonale resulting from multiple infarcts were observed.

\section{Radiographic Features of Pulmonary INFARCTION}

INCIDENCE AND DISTRIBUTION OF RADIOGRAPHIC AbNormalities. - The first part of Table II shows the distribution of the radiographic changes in respect of the side or sides involved. Right-sided involvement alone was commoner than left-sided involvement alone, but bilateral involvement was

TABLE II

INCIDENCE AND DISTRIBUTION OF RADIOGRAPHIC

A. Radiographic Changes.

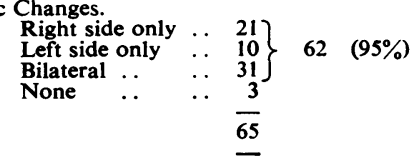

B. Distribution of 117 Opacities in 65 Incidents

$\begin{array}{cccr}\text { Right Side } & \ldots & \mathbf{6 8} & \\ \text { Upper zone } & \ldots & \ldots & 6 \\ \text { Middle zone } & \ldots & \ldots & 12 \\ \text { Lower zone } & \ldots & 49 & \mathbf{5 0} \\ \text { Left side } & \ldots & 49 & \\ \text { Upper zone } & \ldots & \ldots & 4 \\ \text { Middle zone } & \ldots & \ldots & 8 \\ \text { Lower zone } & \ldots & \ldots & 37\end{array}$


the commonest of all ( 31 of 65 incidents). In three cases no changes were observed.

The second part of the table shows the distribution of 117 opacities in the 65 incidents according to the zone involved. The term "zone" is used because lateral films were not available in all cases. Some of these opacities were made up of two or even three separate elements, which will be analysed in Table III, but here only the zonal distribution of the opacities is considered. If more than one type of opacity was present in one zone, only one was counted.

The right lower zone, as in most other recorded series, was the commonest situation for pulmonary infarcts and the left lower zone came next in order of frequency. Infarcts were much less common in the upper and mid zones.

Nature of the Radiographic Abnormalities IN Pulmonary Infarction.-It is possible to classify most of the opacities seen as either of pulmonary or pleural origin. There is little difficulty in recognizing pleural opacities due to effusion or thickening from their characteristic appearance and distribution. Such opacities were present in many cases of this series. A typical example is shown in Fig. 1.

Homogeneous or patchy opacities of nonpleural, presumably intrapulmonary, distribution constitute the second common type of radiographic abnormality. Although, occasionally, opacities of this type correspond in position and shape with atelectatic segments the vast majority do not and are assumed to be produced by the pulmonary infarcts themselves. This interpretation has been confirmed in several cases of the present series at necropsy. For the purposes of description this type of opacity will be referred to as a "pulmonary opacity." It resembles that found in pneumonia and may have either a segmental or a multilobular distribution. Fig. 2 shows bilateral pulmonary opacities due to infarction.

The third type of abnormality, which will be referred to as a "linear opacity," is perhaps the most characteristic of all (Figs. 1, 3, and 4). This term has been applied to horizontal or oblique lines which at first are thick, but which later become thin and sharply defined. In many of the present cases the linear opacity was noted within 24 hours of the first symptoms, but usually it did not appear until after the third or fourth day. Usually it disappeared within two to three weeks, sometimes in less than one week, but occasionally it persisted for several months.
The sharply defined triangular opacity which $\underset{\overrightarrow{\vec{F}}}{\vec{\Rightarrow}}$ was once said to be a common feature of pul=monary infarction was not observed in any case in this series.

Another radiological abnormality commonly疋 found in these cases of pulmonary infarction was an alteration in the position and function of the diaphragm (Figs. 3 and 4). The commonest $\overrightarrow{0}$ feature in the acute stage was a difference in levelbetween the two domes, with inhibited movement of the higher dome. This abnormality is often observed in pleurisy from other causes, but we have gained the impression, which remains to be confirmed by further investigation, that the elevation of the hemidiaphragm with pulmonary infarction is greater and of longer duration than with pleurisy from other causes. True paralysis of one hemidiaphragm, with paradoxical move-⿳亠丷⿵冂丶

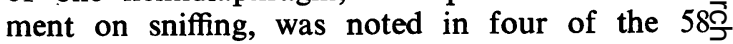
cases. Although this can occur with pleurisy from $\rightarrow$ other causes the incidence must be very muchô lower than in this series of cases of pulmonary infarction. In none of the four cases has diaphragmatic function so far returned. Diaphrag-s matic adhesions were observed frequently after⿳亠二口 recovery from pulmonary infarction.

The radiographic features observed in thiso series of cases of pulmonary infarction may be summarized as follows: (a) Pleural opacities,, 3 (b) pulmonary opacities resembling those found with pneumonic or bronchopneumonic con? solidation, (c) linear opacities, $(d)$ elevation of hemidiaphragm with inhibited movement or음 occasionally, true paralysis, $(e)$ the frequently bilateral distribution of lesions.

It must be emphasized that the individual types of radiographic abnormality encountered ino pulmonary infarction are themselves non-specific? Each one of them can occur, for example, ins cases of pneumonia with pleurisy. The claim however, is made that the coexistence of two or more of these abnormalities, particularly if the changes are bilateral, constitutes a radiologica? picture which gives strong support to a clinica 5 diagnosis of pulmonary infarction. The picturew often alters rapidly either as a result of changes in existing opacities, particularly in linear shadowso or from the appearance of new opacities due toD further infarction. Radiographs repeated at intervals of four or five days after pulmonaryo embolism are therefore helpful in diagnosis.

INCIDENCE OF VARIOUS TYPES OF RADIOGRAPHIC ABNORMALITY.-Table III shows the frequency of each of the three main types of opacity. One hundred and forty-nine separate opacities wer 8 


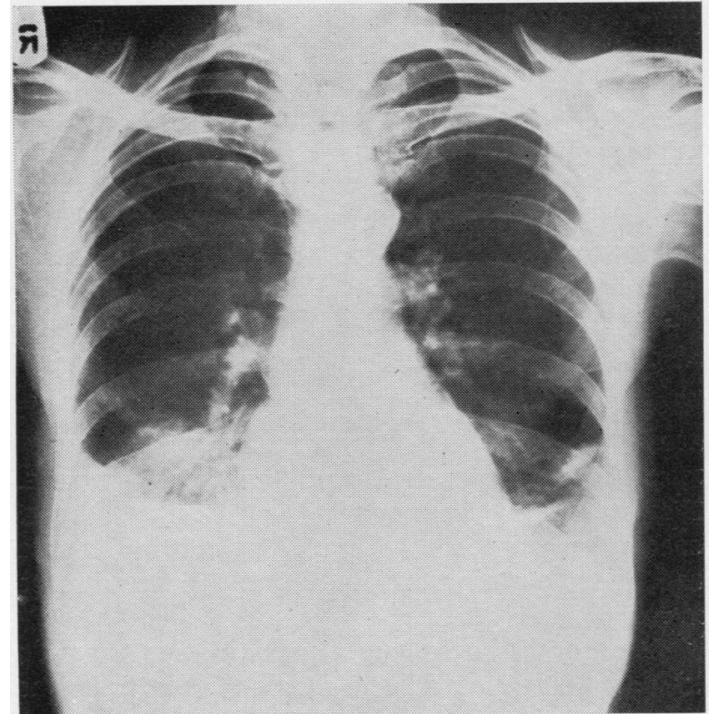

Fig. 1.-Bilateral pleural opacities; pulmonary opacity right lower zone and linear opacities left lower zone.

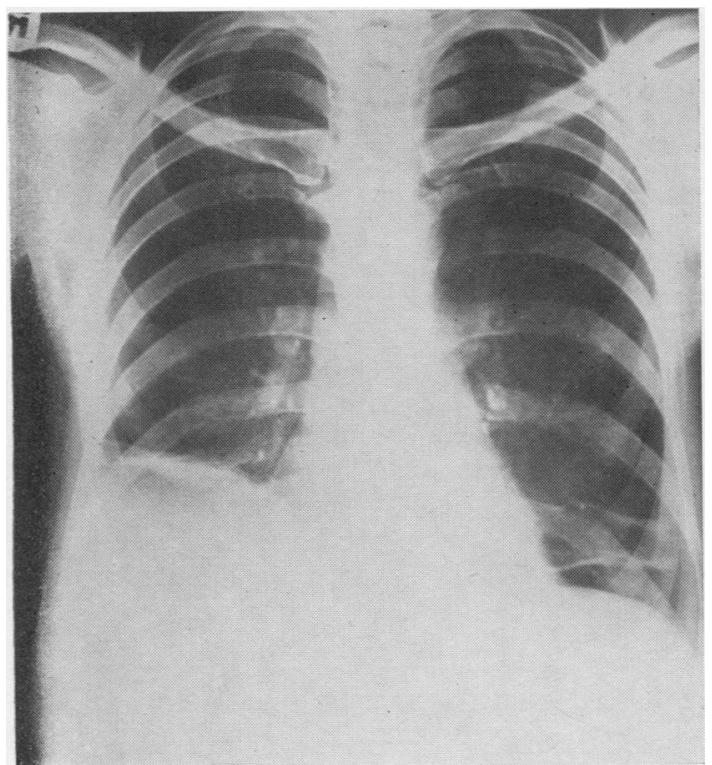

FIG. 3.-High right diaphragm; bilateral linear opacities.

observed in the course of 65 incidents. Their relative frequency differed in cases with heart disease as compared with cases without heart disease. In the cardiac group pulmonary and pleural opacities were frequently encountered. Possibly in some cases the pleural opacity was due not to the effects of pulmonary infarction but to

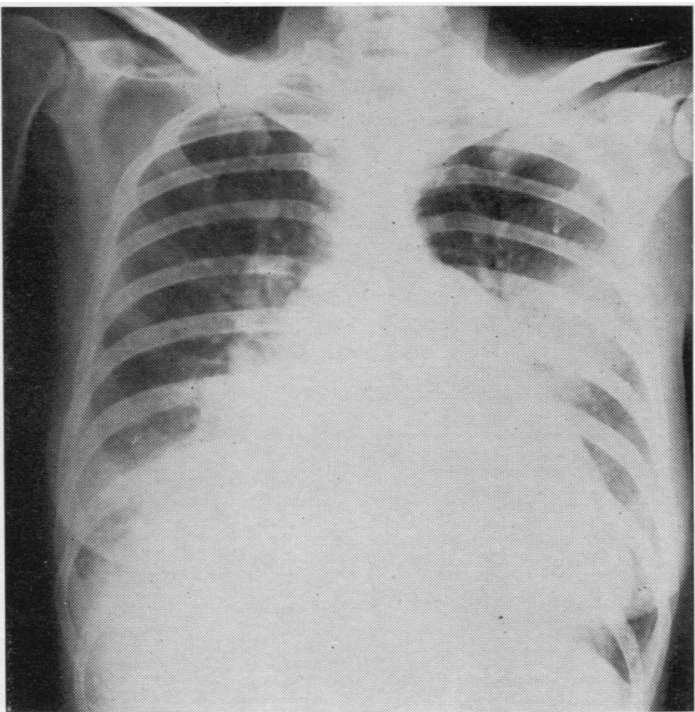

FIG. 2.-Bilateral pulmonary opacities resembling consolidation.

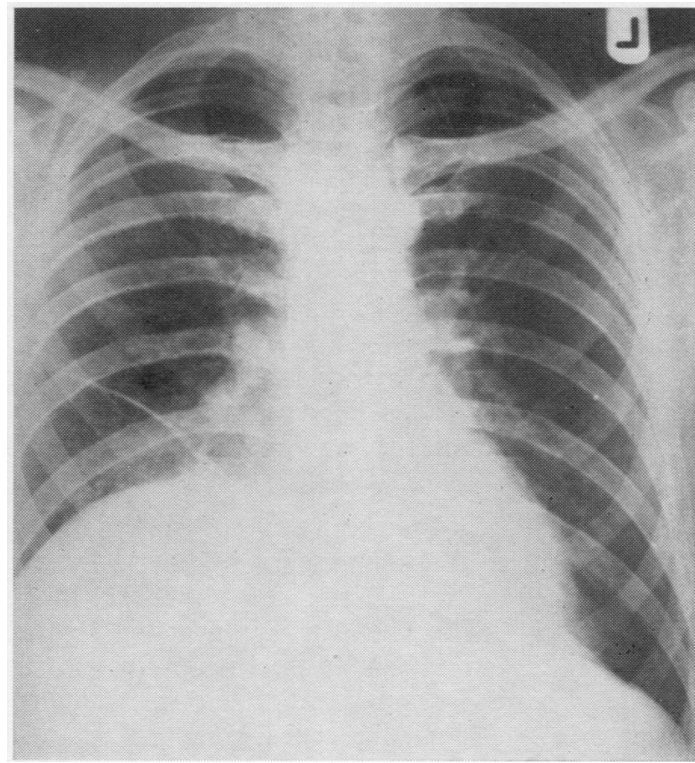

FIG. 4.-High diaphragm and linear opacity on right side.

a small pleural transudate secondary to cardiac failure. Linear opacities were uncommon in this group of cases.

In the non-cardiac group the position was different in that linear opacities were found as frequently as pleural opacities and more frequently than pulmonary opacities. It is not 
clear why this should be so, but a possible explanation is that the pathological nature of what is regarded clinically as pulmonary infarction is in some way different in the cardiac and noncardiac groups.

A hemidiaphragm was definitely elevated in almost $50 \%$ of all incidents of pulmonary infarction. It was not possible to draw any reliable conclusion regarding the relative frequency of this abnormality in the cardiac and non-cardiac groups.

The figures in the present series may be contrasted with those of Short (1951), who claimed to demonstrate an "infarct shadow" in $88 \%$, pleurisy in $56 \%$, and elevation of the diaphragm in $39 \%$. Short included what we have called "pulmonary opacities" and "linear opacities" under the heading of infarct shadows.

TABLE III

NATURE OF 149 SEPARATE OPACITIES IN 65 INCIDENTS

\begin{tabular}{|c|c|c|c|c|}
\hline & & All Cases & $\begin{array}{c}\text { Cases with } \\
\text { Heart } \\
\text { Disease }\end{array}$ & $\begin{array}{c}\text { Cases without } \\
\text { Heart } \\
\text { Disease }\end{array}$ \\
\hline $\begin{array}{l}\text { Pulmonary opacity } \\
\text { Pleural opacity .. } \\
\text { Linear opacity .. }\end{array}$ & $\begin{array}{l}\cdots \\
\ldots\end{array}$ & $\begin{array}{l}47(31 \%) \\
59(40 \%) \\
43(29 \%)\end{array}$ & $\begin{array}{l}14(41 \%) \\
18(53 \%) \\
2(6 \%)\end{array}$ & $\begin{array}{l}33(28 \%) \\
41(36 \%) \\
41(36 \%)\end{array}$ \\
\hline
\end{tabular}

Rate of Clearing of Radiographic Opacities. - The average time taken for resolution in cases with heart disease (48 days) was more than double that in cases without heart disease ( 23 days). Fatal cases and cases in which a complete series of radiographs was not obtained have been excluded from this analysis.

In many cases of the non-cardiac group radiographic resolution was extremely rapid, complete clearing of the opacity or opacities taking place in less than a week.

Clinical and Radiographic Features of CARDIAC AND Non-CARDIAC GRoups.-There were three clear differences between those two groups of cases of pulmonary infarction. (1) Haemoptysis was commoner in the cardiac group if pulmonary congestion was present. (2) Linear opacities were much commoner in the non-cardiac group. (3) Clearing of the radiographic opacities was more rapid in the non-cardiac group.

\section{Differential Diagnosis of Pulmonary INFARCTION}

Pulmonary infarction may be mistaken for many other diseases. In this series the initial diagnosis included pulmonary tuberculosis, primary pleural effusion, pneumonias of various types, lung abscess, and bronchial carcinoma. Ito may be difficult to distinguish pulmonary infarc:0 tion from post-operative atelectasis. In the latter condition phlegm is present though it may not be produced, pleuritic pain is not prominent, and the radiographic opacities, which have a segmental? distribution, usually clear promptly after postura $\vec{P}$ coughing or bronchoscopic aspiration.

\section{Prognosis of Pulmonary Infarction}

The total mortality in the series was $21 \%$ There was no striking difference in mortality between the post-operative and "medical" groups.There was a higher mortality in cases with hearf disease than in cases without, but this differencewas almost certainly related to the heart disease per se.

Acute cor pulmonale occurred in two cases in? which the small embolism causing infarction was later followed by a massive embolism. Both these ${ }^{\curvearrowright}$ patients died.

Five cases developed pulmonary suppuration presumably as a result of secondary bronchogenic infection of infarcted lung, and of these case four died. Three of the five patients, the one whoD survived and two of those who died, developed empyema as a further complication. These cases illustrate the importance of effective chemotherapys as a prophylactic measure in the treatment of pulf monary infarction.

While many cases had small localized pleura $\overrightarrow{\vec{b}}$ opacities, in only three did a large pleura effusion develop. In these cases the effusion took some months to absorb and there was considerable residual pleural thickening, but the patients all survived. Pulmonary infarction should be kepE in mind as an occasional cause of a large chronico pleural effusion.

Six cases, all with pre-existing heart disease developed congestive cardiac failure following pulmonary infarction, and of these six cases fouro died.

\section{Massive Pulmonary Embolism}

Two fatal cases of massive pulmonary embolisnt? were studied clinically and pathologically. Onę patient died 10 hours after the embolism, whiles the other survived for six weeks after the onset of symptoms. They each presented a number of instructive features relating to the effects of pul-ब monary embolism.

CASE 1.-A woman, aged 59 years, suffering from thrombo-phlebitis, developed a massive pulmonary embolism. A radiograph of the chest was obtained nineo 
hours after the initial symptoms and one hour before death. It showed that the vascular markings of the right lung were distinctly less prominent than those of the left, and that the markings on the left were less prominent than on a routine radiograph taken six weeks previously. In addition, there was marked distension of the superior vena cava. At necropsy a coiled embolus totally occluded the lumen of the right pulmonary artery and partially obstructed the left pulmonary artery. The lungs themselves appeared normal both macroscopically and microscopically.

This case illustrates the radiographic phenomenon first described by Westermark (1938), namely, relative ischaemia of a lung or part of a lung distal to an embolism. He also included in his description a dense pulmonary artery shadow with a sharp distal termination, presumably representing the embolus in situ.

CASE 2.-A man, aged 36, was admitted to hospital two months after internal saphenous ligation for varicose veins, with a two weeks' history of increasing breathlessness on exertion which had started suddenly after a short period of central chest pain.

On admission he was orthopnoeic, deeply cyanosed, and mildly febrile. The heart rate was 84 per minute and the rhythm regular; there was no clinical evidence of cardiac enlargement and no peripheral oedema, but gallop rhythm was present and the external jugular veins were distended. Radiography of the chest five days before admission showed prominence of both hilar shadows. The lower branch of the right pulmonary artery terminated abruptly at the lower end of the hilum without branching in the normal manner, and only a few spidery vascular markings were visible in the lower zone of the right lung. The markings in the left lower zone were also less prominent than normal. The reduction in the vascular markings in the lower lung fields resembled that found in emphysema. An electrocardiogram showed an abnormal pattern of the type associated with myocardial ischaemia.

Despite treatment with anticoagulants and digitalis, the breathlessness increased and gross peripheral oedema developed. A radiograph three weeks after admission showed marked cardiac enlargement, mainly right sided with prominence of the pulmonary trunk, in addition to the abnormalities noted in the previous film. Five days later he had a severe attack of upper chest pain, situated mainly to the left of the mid-line and not related to rispiration, which was followed by a further increase in dyspnoea and cyanosis. He died on the following day.

At no time during the illness were crepitations heard on auscultation of the lungs. A further electrocardiogram, taken a few hours before death, showed changes compatible with acute right ventricular strain.

At necropsy the right and left pulmonary arteries and their larger branches were all completely occluded by firm, adherent thrombus.

The lungs were emphysematous and there was an early infarct in the right lower lobe. There was gross dilatation and some hypertrophy of the right ventricle and of the right atrium. Extensive venous thrombosis was found in the lower limbs.

This patient presented the clinical, radiographic, electrocardiographic, and pathological features of subacute cor pulmonale which was considered to be the result of progressive thrombosis throughout the pulmonary arterial tree initiated by a massive embolism. Despite the severity of the vascular occlusion, pulmonary infarction was not a conspicuous feature. Some of the radiographic features of pulmonary embolism described by Westermark were present, but these could equally well have been produced in this case by emphysema.

\section{AN INTERPRETATION OF THE}

\section{PATHOLOGICAL AND OTHER PROCESSES} FOLLOWING PULMONARY EMBOLISM

In considering the effects of pulmonary embolism attention must first be paid to the size of the embolus. Gibbon, Hopkinson, and Churchill (1932) have demonstrated in experimental animals that $60 \%$ of the total pulmonary circulation must be obstructed before the blood pressure falls and the right ventricle is embarrassed, and probably $80 \%$ before death is likely. It is after such massive emboli that acute cor pulmonale is most frequently encountered. The present series included four cases of this type. In two of them the fatal embolism was preceded by pulmonary infarction due to smaller emboli.

A less massive embolus may lodge near the bifurcation of the pulmonary trunk and cause acute dyspnoea, circulatory collapse, or central pain simulating myocardial infarction and probably due to myocardial ischaemia. These symptoms may be relieved if the embolus fragments and passes on to the right and left pulmonary arteries. This may then result in bilateral infarction with pleuritic pain, as occurred in one of our cases. Smaller emboli usually produce pulmonary infarction without any preceding acute circulatory disturbance.

In this as in other series the right lower lobe and the left lower lobe were in that order the most frequent sites of infarction. This distribution of embolic lesions has been reproduced in experimental animals (Businco and Cardia, 1931) by the injection of bismuth into a femoral vein. Subsequent fluoroscopic examination of the lungs showed the opaque medium filling the right lower lobe first and then the left lower lobe before being distributed to the rest of the lungs. This has been explained as being related to the direction, length, width, and distribution of the right pulmonary artery.

In many of our cases the infarction was bilateral. The probable explanation of this is that 
the clot, which must be friable by the very reason of its detachment, is liable to disintegrate on its way to the lungs. The diagnostic importance of these bilateral lesions has already been emphasized.

\section{Pulmonary Infarction}

Embolism without infarction is well recognized in experimental animals in which it is very difficult to induce infarction except in the presence of pulmonary congestion. In some cases of pulmonary embolism in man there may be no infarction. This occurs if the embolus does not wholly occlude the vessel, as when a coiled embolus lodges in a large pulmonary artery, or if the collateral circulation is adequate to prevent infarction. In most cases, however, embolism is followed by infarction. The lesions produced vary greatly in extent and severity from case to case.

\section{HAEMORRHAGIC INFARCTION}

In this, the most severe type of pulmonary infarction, the histological appearance is that of haemorrhagic necrosis (Figs. 5 and 6). In one area of Fig. 6 the alveolar walls can just be detected, but even here it is clear that they have been damaged beyond repair. In the present series when infarcts of this type were demonstrated at necropsy, generalized pulmonary congestion was present in most instances.

\section{INCOMPLETE INFARCTION}

In haemorrhagic infarction the alveoli are destroyed and repair takes place by organization. Such a slow fibrotic process is not compatible with the variable and transitory radiographic findings in the majority of cases of this series. There was rarely any residual scar demonstrable such as would be expected if a haemorrhagic infarct had occurred and healed by fibrosis. Although experimentally it is difficult to produce haemorrhagic infarction in normal lungs, clinical and radiographic studies such as this show that some change does occur in the healthy human lung as a result of embolism. Pathological material to demonstrate this process is difficult to obtain as these patients generally survive. Hampton and Castleman (1940) drew attention to certain lesions which occurred as a result of embolism in experimental work carried out in animals with healthy lungs. These were not regarded as true infarcts because there was no destruction of the alveolar wall. There was, however, local pulmonary congestion and oedema with some haemorrhage into the alveoli. Identical findings were also demonstrated in a woman who had a post-operative infarct and who died "a few days later" from massive pulmonary embolism. To these changes Hampton and Castleman applied the term "incomplete infarction."

In the pathological material available in the present series examples of incomplete infarction is were readily found. Figs. 7 and 8 show what is $\overrightarrow{0}$ considered to be the earliest stage of incomplete infarction, i.e., localized pulmonary congestion. $\vec{\omega}$ It is seen that this is most marked in the pleura $\vec{\sim}$ and subjacent lung. In contrast with true $x$ haemorrhagic or "complete" infarction seen in Fig. 5 the alveolar walls are intact.

A lung section from the patient (Case 2) who had bilateral pulmonary emboli but no macro- $\triangle$ scopic evidence of infarction shows congestion $\vec{z}$ and considerable oedema (Fig. 9), which can be

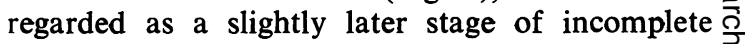
infarction. A similar example is shown in Fig. 10. This patient had widespread pulmonary infarction of demonstrated radiographically; shortly before : death this was clearing dramatically following the relief of generalized pulmonary oedema by mercurial diuretics. At necropsy only one infarcted area was found; this showed localized congestion and oedema and was presumably capable of $\stackrel{\mathbb{Q}}{\mathscr{Q}}$ resolution.

A more advanced stage of incomplete infarction, where in addition to congestion and oedema there is alveolar haemorrhage, is seen in Fig. 11. The alveolar walls, however, have the appearance of being viable. Haemoptysis is to be expected in a lesion of this type but not in the previous $\underset{x}{\stackrel{0}{x}}$ examples of incomplete infarction. Fig. 12 shows $\dot{0}$ a late stage of incomplete infarction, the changes of which may or may not be reversible. At this stage incomplete infarction merges into the complete. The pathological changes in pulmonary 윽 infarction thus range from congestion to oedema, $>$ alveolar haemorrhage, alveolar wall destruction, and complete haemorrhagic necrosis. In almost every case of complete infarction in this series $\sigma$ study of adjacent areas of lung showed all degrees $\tilde{\sim}$ of incomplete infarction.

Pleural Changes in Pulmonary Infarction

Clinical, radiographic, and pathological evidence $\overparen{\mathbb{D}}$ all suggest that in infarction the pleura and $\stackrel{\mathcal{+}}{+}$ adjacent lung are predominantly involved. Pleur- 70 itic pain is the commonest symptom. In the survey of radiographic findings it was concluded $\stackrel{\Phi}{\Phi}$ that the changes most frequently seen are pleural $\stackrel{\mathbb{Q}}{\varrho}$ in origin. Hampton and Castleman have shown that the pleura is always involved, and by careful 


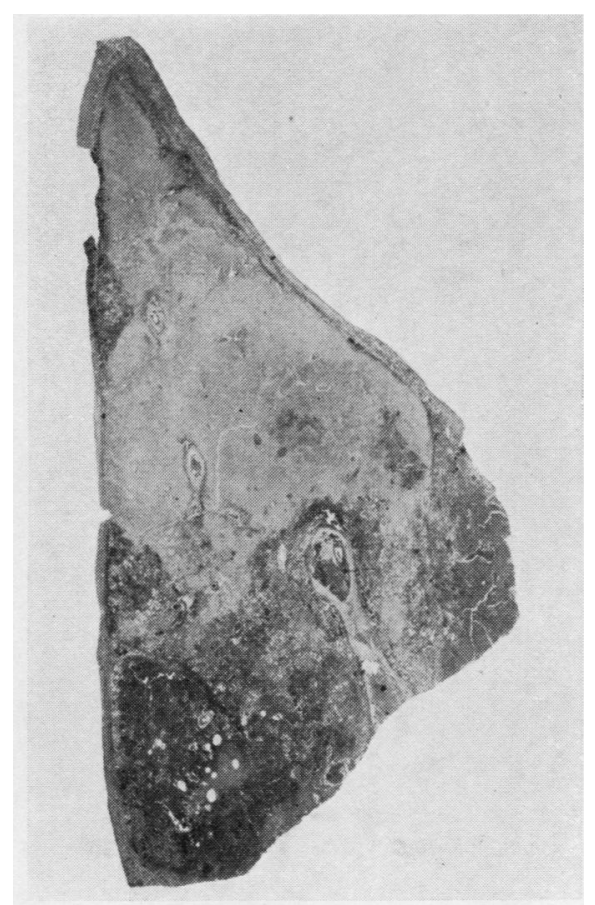

Fig. 5.- $\times$ 3. True haemorrhagic or complete infarction seen best at the top of the section. The lowest dark area is an artefact. ,

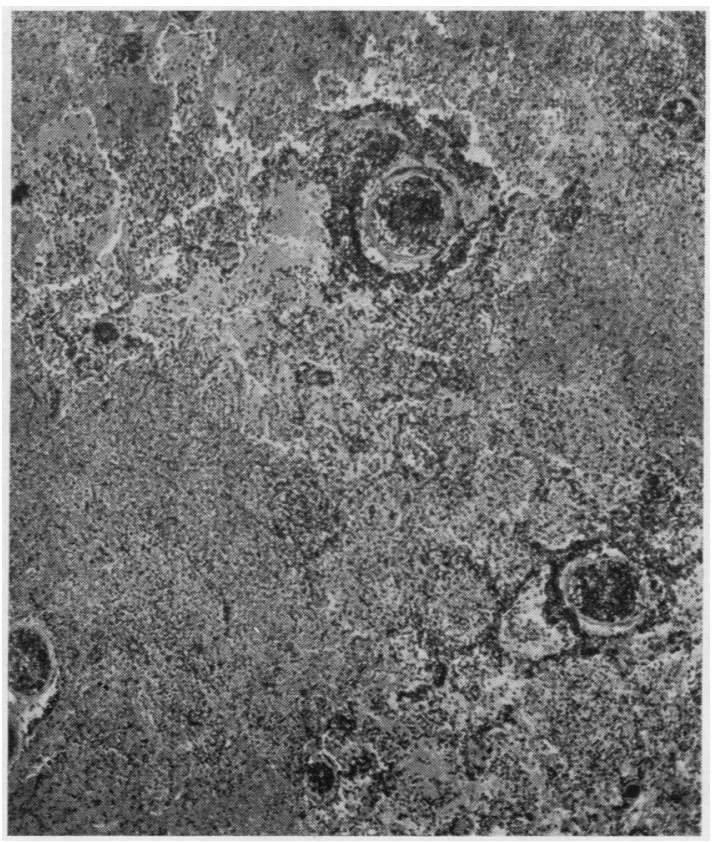

FiG. 6. $-\times 50$. High power view of Fig. 5, showing extensive destruction of the alveolar walls.

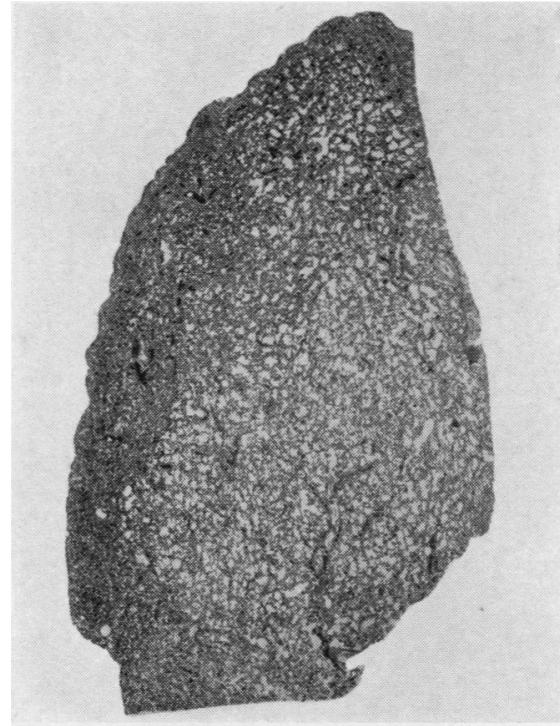

FIG. 7. $-\times 3 . \quad$ Incomplete infarction showing congestion of pleural and sub-pleural areas.

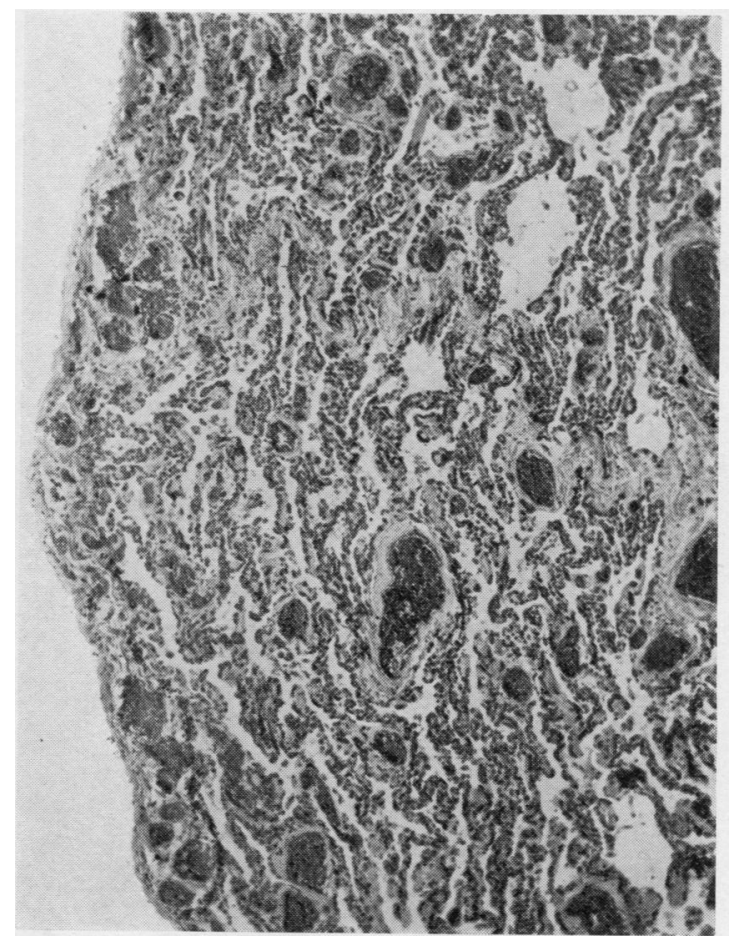

Fig. 8. $-\times 50$. High power view of Fig. 7, showing congestion of sub-pleural areas. 


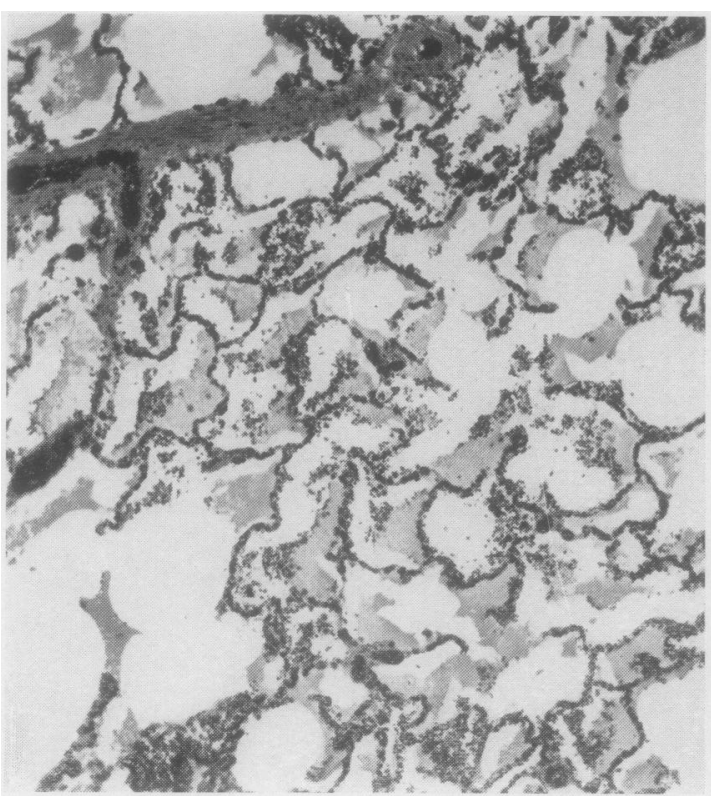

Fig. 9. $-\times 50$. Incomplete infarction showing congestion and oedema.

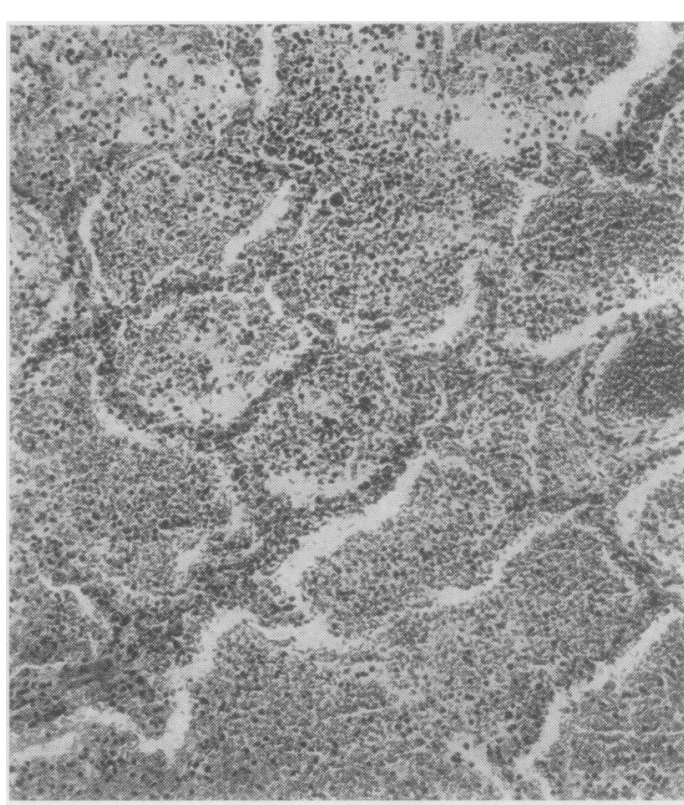

FIG. 11. $-\times 100$. Incomplete infarction showing alveolar haemor-ō rhage.

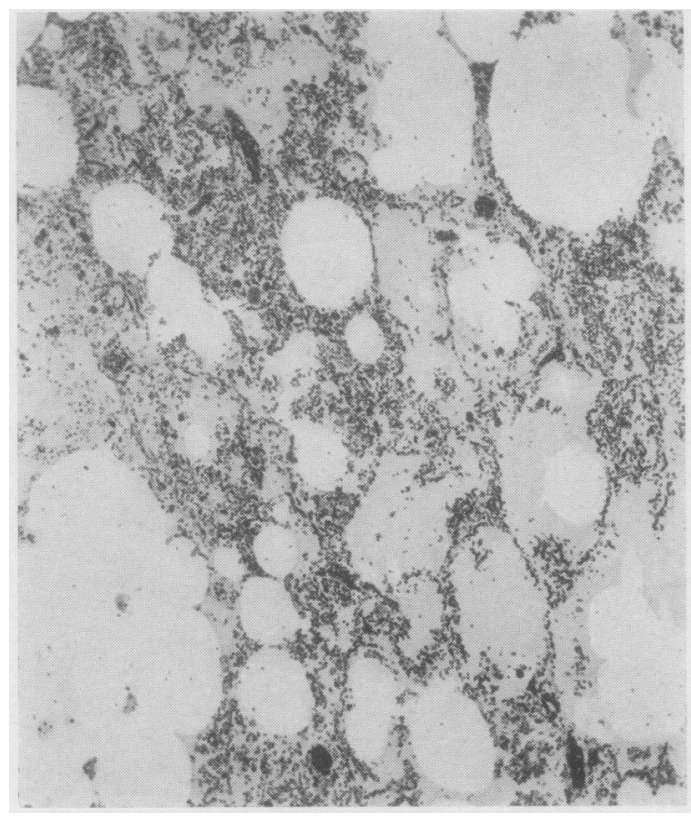

FIG. 10. $-\times 50$. Incomplete infarction showing congestion and oedema.

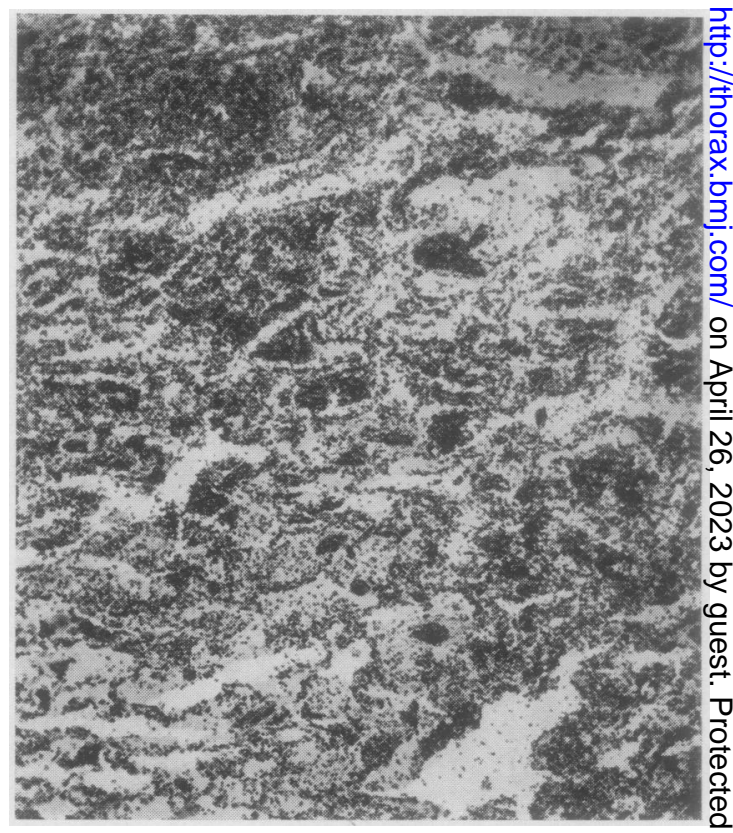

FIG. 12. $-\times 50$. Late stage of incomplete infarction showing alveolar wall destruction.

\section{을 \\ 을}


dissection of infarcts have demonstrated that the longest axis of the infarct is parallel to the longest pleural surface involved. In the earliest stages of incomplete infarction the congestion is most marked in the sub-pleural areas (Fig. 7). A possible explanation of this is that the degree of ischaemia is more severe at the periphery of the lung, where the collateral circulation is likely to be less efficient.

\section{Circulatory Adjustments in Complete and INCOMPLETE INFARCTION}

Until recently the experimental evidence regarding the relative importance of the bronchial and pulmonary circulation in the production of infarction has been conflicting. Ellis, Grindlay, and Edwards $(1951,1952)$ have done much to clarify the position by experiments in dogs using new techniques which appear reliable. They demonstrated that the bronchial artery to a lobe could be occluded without resulting pathological changes in that lobe, and concluded that the pulmonary arterial circulation supplied the nutritive requirements of the lung beyond the hilum. Occlusion of a branch of the pulmonary artery as well as the bronchial artery produced no pathological change, i.e., an adequate collateral circulation was provided by pulmonary capillaries. They showed, however, that when the bronchial circulation was intact emboli resulted in complete and incomplete infarcts, and that the amount of haemorrhage varied with the degree of dilatation of the bronchial vessels. Other workers have shown that there is an enormous dilatation of the bronchial arteries beginning a few days after infarction. Ellis therefore makes two main claims: (1) The pulmonary arteries provide the collateral blood supply after infarction, and (2) the haemorrhage in infarction comes from the bronchial arteries. With this work in mind we suggest the following scheme (see next column) as compatible with the pathological changes.

While it is believed that the interpretation given applies to embolism throughout the greater part of the pulmonary tree, it may not apply to embolism involving a major pulmonary artery. Here broncho-pulmonary arterial anastomoses may alter the circulatory adjustments (Ellis and others, 1952).

Complete infarction is particularly liable to occur when there are adverse circulatory factors such as generalized pulmonary congestion. This concurs with the repeated observation that in animals pulmonary congestion is a prerequisite for true infarction. In the present series it has been
OCCLUSION OF PULMONARY ARTERY BY EMBOLUS

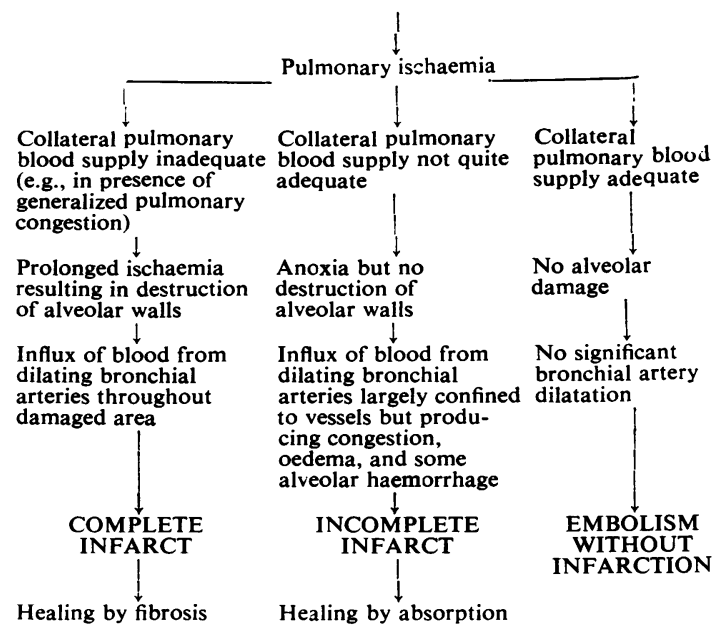

demonstrated that infarction in patients with heart disease runs a longer course compared with infarction in healthy lungs ; this is presumably because most of these cases had complete infarcts.

It is suggested that incomplete infarction resulting in local congestion and oedema of lung and pleura gives a satisfactory explanation of the outstanding clinical and radiographic features of embolism in healthy lungs. Complete infarction, though it may occur in healthy lungs, is much more common if the pulmonary circulation is inefficient. If this is true it is obvious that, in the treatment of pulmonary infarction, particular attention must be paid to the relief of any generalized pulmonary congestion.

\section{Correlation of Clinical and Pathological FINDINGS}

Pulmonary Opacities.-In several cases of the present series opacities of this type were shown at necropsy to have been produced by the infarct itself. It has been clearly demonstrated by many previous workers that the infarct shadow is not necessarily triangular in shape. This point, however, is worthy of further emphasis. The infarct shadow is often ill defined, as indeed would be expected if it reflects local congestion and oedema alone or bordering on an area of complete infarction. We have often been impressed by the contrast between the radiographic evidence of extensive pulmonary involvement and the small macroscopic infarct found at necropsy. The explanation suggested is that around the area of destruction there is extensive congestion and oedema, i.e., the changes of incomplete infarction surround the complete infarct. In cases with 
heart disease the infarct shadow is more clearly defined as would be expected when complete infarction predominates.

Pleural Opacities.-In most cases these must represent small pleural effusions or areas of fibrinous exudate overlying a pulmonary infarct.

Linear OPacities.-It is suggested that these are produced by sharply localized lesions of the pleura and subjacent lung. This explanation is in keeping with the observation that linear shadows are more commonly associated with pleural than with pulmonary opacities. Of a total of 43 linear opacities in the present series, 37 were accompanied by pleural opacities on the same, opposite or both sides ; in contrast only six of 43 linear opacities were associated with pulmonary opacities. As there was little difference between the total numbers of pleural and pulmonary opacities in the series these figures suggest that both the linear and pleural opacities may well be manifestations of the same process, viz., pleural congestion, oedema and exudation, and that the linear opacity represents a localized pleural reaction over the site of a pulmonary infarct which may itself not be visible. A further point in favour of a pleural origin for linear opacities is that in several cases a pleural opacity was observed to change into a typical linear opacity.

It has not been possible to obtain with certainty a histological section of a linear opacity. This is probably because the linear opacity is usually a transitory phenomenon and occurs most frequently in non-fatal cases. Occasionally, however, a linear shadow does not resolve. Hampton and Castleman were able in one such instance to correlate the clinical, radiographic, and necropsy findings. They demonstrated convincingly that this linear opacity was due to pleural thickening which had proceeded to fibrosis. Hampton and Castleman were reluctant to accept a pleural origin for all linear opacities and suggested the alternative of discs of atelectasis. Short (1951) also supported this view. It is difficult to see why atelectasis should occur when there is no pathological evidence of bronchial or bronchiolar obstruction and when the production of phlegm in infarction is usually inconspicuous.

The above evidence of a pleural origin for linear opacities is as yet incomplete, but the problem is being subjected to further study. If a pleural origin for linear shadows is accepted the relative infrequency of such shadows in patients with heart disease may be related to the complete infarction which usually occurs in this type of case, the pleural shadow being obscured by the pulmonary shadow.

Diaphragmatic Changes.-(a) Immobility of 흠 the diaphragm is probably reflex in origin and $\frac{\bar{S}}{D}$ associated with the pleuritic pain. (b) Paradoxical $\overparen{D}$ movement was seen in four cases, and was apparently permanent. Its cause is unknown. It is $\overrightarrow{0}$ conceivable that the phrenic nerve trunk is involved in a pleural reaction. Possibly the $\vec{\omega}$ branches of the nerve as they enter the diaphragm are damaged by pleurisy. This may be one $\overrightarrow{\vec{x}}$ explanation of so-called "idiopathic" diaphrag- 0 matic eventration, i.e., an end-result of pulmonary embolism. (c) Local impairment of diaphragmatic $\underset{ }{ }$ movement occurs as a result of pleural adhesions.

\section{- SUMMARY}

The clinical and radiographic features of 60 cases of pulmonary embolism are presented; of these, 58 had pulmonary infarction and two had $\vec{\emptyset}$ massive pulmonary emboli without infarction.

After pulmonary embolism, there may be no infarction, incomplete infarction, or complete infarction. Consideration is given to the parts played by the pulmonary and bronchial circulations in these events.

Incomplete infarction is characterized by localized congestion and oedema of the lung and pleura proceeding in some instances to intraalveolar haemorrhage. This is the manifestation of embolism most frequently encountered in healthy lungs.

Complete or haemorrhagic infarction is characterized by necrosis of the alveolar walls and is most $\frac{x}{0}$ commonly found in the presence of generalized pulmonary congestion.

The radiographic appearances are described as pulmonary, pleural, and linear opacities, and $\mathrm{O}$ diaphragmatic abnormalities. The right lower zone is most commonly involved. The distribution of the radiographic lesions is frequently bilateral. Linear opacities are frequently associated with $\tilde{N}$ pleural opacities and are much commoner in $N$ patients without heart disease. They have ten-N tatively been attributed to pleural and subjacent ${ }_{\sigma}^{\omega}$ pulmonary oedema rather than to plaques of atelectasis. The resolution of the radiographic changes is more rapid in healthy lungs than in the presence of generalized pulmonary congestion. This has been attributed to the higher incidence of incomplete infarction in the former circum- $-\bar{\Phi}$ stances, and of complete infarction in the latter. $\frac{\text { ते }}{\mathbb{C}}$ Radiographs repeated at intervals of four or five days are valuable in the diagnosis of pulmonary? infarction. 
Bronchogenic pulmonary suppuration is a common and often fatal complication. Pulmonary infarction may cause a large, persistent pleural effusion or may precipitate cardiac failure. Permanent paralysis of a hemidiaphragm may follow pulmonary infarction.

In treatment prophylactic chemotherapy and measures directed to the relief of generalized pulmonary congestion are important.

The diverse effects of pulmonary embolism are explained by the variation in the size and number of the emboli, by the frequently bilateral distribution of the resulting infarcts, by the presence or the absence of pulmonary congestion before the embolism, by the type of infarct that develops, complete or incomplete, and by complications such as infection.

The authors are indebted to their colleagues in the Northern Group of Hospitals, Edinburgh, who so willingly and freely gave them access to patients, and to Professor L. S. P. Davidson, Professor John Crofton, and Dr. R. W. D. Turner for much helpful criticism.

REFERENCES

Businco, O., and Cardia, A. (1931) Fortschr, Röntgenstr., 44, 60

Ellis, F. H., Jr., Grindlay, J. H., and Edwards, J. E. (1951), Surgery $30,810$. (1952). Ibid., 31, 167.

Gibbon, J. H., Hopkinson, Mary, and Churchill, E. D. (1932). J. clin. Invest., 11, 543

Hampton, A. O., and Castleman, B. (1940). Amer. J. Roentgenol. 43, 305

Short, D. S. (1951). Ouart. J. Med., 20, 233.

- (1952). Brit. med. J., 1, 790.

Westermark, N. (1938). Acta radiol., Stockh., 19, 357. 